\title{
Autowave nature of plasticity. Scale invariance
}

\author{
Lev Zuev ${ }^{1, *}$ \\ ${ }^{1}$ Institute of Strength Physics and Materials Science, SB RAS. Tomsk
}

\begin{abstract}
The generality of localization of plastic deformation, which is observed at the stage of linear work hardening for HCP, BCC and FCC mono- and polycrystals of pure metals and alloys, is considered. It was found previously that the motion rate of localized flow autowave is related to the reciprocal value of the work hardening coefficient by a linear law, which is universal in character. This is further substantiated by the results of the given study. The waves of plastic flow localization are found to have dispersion law. It has been established that in order to address the autowave of localized deformation, a quasi-particle may be introduced. The quasi-particle's characteristics have been defined.
\end{abstract}

\section{Introduction}

Studies of plastic deformation in solids was carried on; the research results were summarized in the monograph [1]. It has been established that the plastic deformation tends to localize on the macro-scale level in any solid from the yield limit to the failure. The localization phenomenon is manifested as spontaneous layering of the material bulk into non-deformed and actively deforming nuclei, which are arranged over the test sample, making up a clearly defined pattern. Each pattern is in close correspondence with the law of deformation hardening acting at the given flow stage. The order of appearance of patterns is as follows: switching autowaves; phase auto-waves; stationary dissipative structures. At the pre-fracture stage collapse of autowaves would occur. The typical example is shown in fig. 1 a.

The emergence of patterns in the deforming medium is evidently due to the selforganization of its defect structure. It is well known that the processes involved in the selforganization of an open medium would result in a decrease in the rate of entropy production $[2,3]$, which suggests that a detailed description of plastic deformation localization processes can be discussed in terms of entropy production rate. This will be discussed and exemplified where necessary in what follows. A more convenient and therefore preferred is the stage of linear deformation hardening where phase autowaves of localized plasticity with the length, $\lambda \approx 10^{-2} \mathrm{~m}$, propagate at the rate $V_{a w} \approx 10^{-5} \ldots 10^{-4} \mathrm{~m} / \mathrm{s}[1]$.

Apparently, all the nuclei of localized deformation are distributed periodically in space; the mobile nuclei will move along the test sample in a concerted manner with a rate $V_{a w}$. It follows from the diagrams $X(t)$ shown in Fig. $1 \mathrm{~b}$ that the condition $V_{a w}=d X / d t=$ const is

\footnotetext{
${ }^{*}$ Corresponding author: $\underline{1 b z @ i s p m s . t s c . r u ~}$
} 
satisfied for all the stages of work hardening, which is significant enough to deserve further discussion.

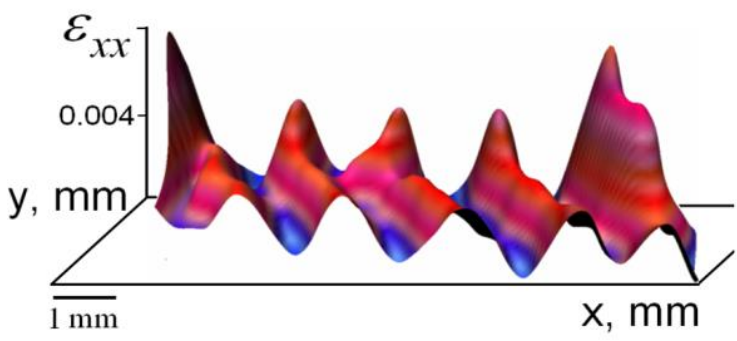

a b

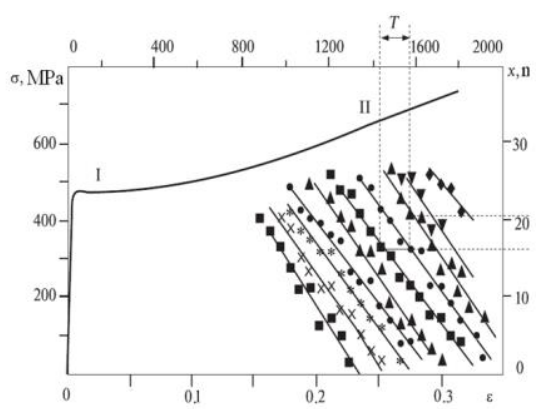

Fig. 1. A typical pattern of localized plastic flow for the case of linear work hardening (a); an explanation for the estimation of autowave length and autowave period with the help of X-t diagram (b)

\section{Autowaves and elastic-plastic strain invariant}

A qualitative analysis of experimental data rests on the observation that the regularities represented in fig. 1 a serve to provide a unified explanation of the plastic flow behavior. The interpretation of experimental evidence is subtle and needs careful explanation. It has been established that to each flow stage corresponds a respective localization pattern; hence, we can set up a one-to-one correspondence between the emergent localization pattern and the respective work hardening stage. A key aspect of this many-faceted problem that will be dealt with here is the nature of localized plasticity. The basic notion is that the above features of localized plasticity patterns are analogous to the well-known phenomena synergetics deals with - namely, dissipative structures called 'autowaves' or 'self-excited waves' [4]. Special variants of these structures, which are also known as 'switching autowaves', 'phase autowaves' and 'stationary dissipative structures', have been studied in detail for chemical and biological systems. However, we were the first to discuss these structures in the context of solids' plasticity problem.

The localized deformation pattern, which emerges at the stage of linear work hardening, is the most intriguing one. Under the condition $\sigma \sim \mathcal{E}$, in all the test specimens, whether single- or polycrystalline, a sequence of local strain domains is found to move synchronously along the specimen extension axis. These domains are equidistant and move at the same constant rate. Therefore, this pattern may be regarded as a specific wave process associated with the plastic deformation.

The quantitative analysis was made of the research results. It was found that the characteristics of phase auto-waves propagating at the stage of linear deformation hardening, i.e. auto-wave length, $\lambda$, and auto-wave velocity, $V_{a w}$, as well as the deforming medium's characteristics, i.e. interplanar distance, $\chi$ and transverse elastic wave rate, $V_{t}$, enter the following dimensionless relation:

$$
\frac{\lambda V_{a w}}{\chi V_{t}}=\hat{Z} \approx \frac{1}{2} .
$$

Relation (1) has been called elastic-plastic strain invariant. It is based on the original data obtained for a range of metals and alloys listed in the Table (note that the values $\lambda$ and $V_{a w}$ are quantitative characteristics of localized plastic deformation, while $\chi$ and $V_{t}$ play the same role for elastic deformation). Hence, relation (1) indicates that elastic and plastic 
deformation processes occurring in the medium are interrelated, which provides additional insights into the kinetics of form changing. Its usefulness in application has to be verified by exploring the nature of invariant (1). This problem can be addressed by testing the hypothesis for the normal distribution of the quantity $\hat{Z}$ for different materials. This hypothesis can be accepted on the condition that linear correlation exists between the values of the variable $\hat{Z}$ on the one hand and the quantiles of normal distribution on the other hand. To establish a correlation between the above two sets, we shall apply a graphical method for plotting a dependence of values of the variable $\hat{Z}$ on quantiles of the normal distribution $Q$, which were tabulated for the probabilities $k=i /(n+1)$ (here $n=19$ is the number of studied metals and alloys; $i=1,2,3 \ldots 19$ ). Calculations have been made which show that the correlation coefficient for the quantities $Q$ and $\hat{Z}$ is $\sim 0.6$, which suggests that the distribution of the invariant $\hat{Z}$ is close to normal. The spread of values $\hat{Z}$ is accountable to inaccurate experimental determination of the values $\lambda$ and $V_{a w}$ for the phase autowaves of localized plastic flow.

The following are theoretical considerations of the physical nature of the invariant, which are based on thermodynamic estimates. The plastic deformation is described in terms of transformation of elastic and plastic strain fields, which occurs in a concerted manner in the deforming solid. The process kinetics is determined by the values which enter invariant (1). The distribution of elastic and plastic strain fields occurs at rates $\sim V_{t}$ and $\sim V_{a w}$, respectively; the characteristic spatial scales of the processes are determined by the values $\chi$ and $\lambda$. Thus, invariant (1) reflects the intricate reciprocal dependence of processes involved in the redistribution of strains and stresses by the plastic flow: due to stress relaxation, deformation would occur, while a change in the deformation level would initiate rearrangement of the elastic stress field.

It is demonstrated in [1] that the autowave phenomenon appearing in the deforming solid is described by parabolic differential equations of rate, which have been derived for variations in the strains and stresses, i.e. $\dot{\varepsilon}=f(\varepsilon, \sigma)+D_{\varepsilon} \varepsilon^{\prime \prime}$ and $\dot{\sigma}=g(\varepsilon, \sigma)+D_{\sigma} \sigma^{\prime \prime}$, respectively (here $D_{\varepsilon} \varepsilon^{\prime \prime}$ and $D_{\sigma} \sigma^{\prime \prime}$ are 'diffusion' type members responsible for the spatial distribution of strains and stresses; non-linear functions $f(\varepsilon, \sigma)$ and $g(\varepsilon, \sigma)$ describe local relaxation acts). The coefficients $D_{\varepsilon}$ and $D_{\sigma}$ have the same dimension as the diffusion coefficient $\mathrm{L}^{2} \cdot \mathrm{T}^{-1}$. In view of the diffusivity of strain and stress redistributions, there is reason to believe that the flows of strains and stresses, i.e. $j_{\varepsilon}=\dot{\varepsilon} / A$ and $j_{\sigma}=\dot{\sigma} / A$ (here $A=1$ is sample cross-section area) are proportional to both the strain and stress gradients, i.e. $\nabla \varepsilon$ and $\nabla \sigma$, respectively; hence, the interdependence of the values $\sigma$ and $\varepsilon$ is taken into account, considering that $\sigma(\varepsilon)$. In view of the fact that the deformation, $\varepsilon$, is the 'key' variable employed for plastic flow description, the following set of equation is obtained:

$$
\left\{\begin{array}{l}
j_{\varepsilon} \sim \dot{\varepsilon}=D_{\mathscr{}} \nabla \varepsilon+D_{\delta \sigma} \nabla \sigma, \\
j_{\sigma} \sim \dot{\sigma}=D_{\sigma \varepsilon} \nabla \varepsilon+D_{\sigma \sigma} \nabla \sigma .
\end{array}\right.
$$

The flows $j_{\varepsilon}$ and $j_{\sigma}$ are interdependent. Onsager's reciprocity principle [5] asserts symmetries for the coefficients from the set of Eqs. $2 a$ and $2 b$; hence, the equality 
$D_{\varepsilon \sigma}=D_{\sigma \varepsilon}$ holds good. By the plastic deformation, structural changes will occur; the variation in the production of entropy $(S)$ in the system changes in concert with the variables $\varepsilon$ and $\sigma$. The pace of variation is formally determined by the derivatives $\partial S / \partial \varepsilon$ and $\partial S / \partial \sigma$. It is possible to propose, the flows $j_{\varepsilon}$ and $j_{\sigma}$ in Eq. (2) are proportional to $\partial S / \partial \varepsilon$ and $\partial S / \partial \sigma ;$ hence,

$$
\begin{aligned}
& \dot{\varepsilon}=\Gamma_{\delta \sigma} \partial S / \partial \sigma, \\
& \dot{\sigma}=\Gamma_{\sigma \varepsilon} \partial S / \partial \varepsilon,
\end{aligned}
$$

respectively. According to Onsager's reciprocity principle [5], the following equality also holds good for the coefficients of Eqs. (3) and (4)

$$
\Gamma_{s \sigma}=\Gamma_{\sigma \varepsilon} \text {. }
$$

From dimensional considerations, it is convenient to write

$$
\begin{aligned}
& \Gamma_{\delta \sigma}=\dot{T} \ddot{u} /\left(\lambda V_{a w}\right)^{2}, \\
& \Gamma_{\sigma \varepsilon}=\dot{T} \ddot{u} /\left(\chi V_{t}\right)^{2},
\end{aligned}
$$

where $\dot{T}$ is the rate of temperature variation and $\ddot{u}$ is acceleration of the rate. The latter two values may be regarded as local characteristics of the deformed material behavior within the plastic deformation nucleus. In this case, $\dot{T}=$ const and $\ddot{u}=$ const ; hence,

$$
\lambda V_{a w} \approx \chi V_{t}
$$

which determines the elastic-plastic strain invariant with precision to the constant $\hat{Z}$. It is thus believed that the elastic-plastic strain invariant is the consequence of the non-linear connection between strains $\varepsilon$ and stresses $\sigma$.

To estimate the magnitude of the value $\hat{Z}$, equation (1) is written as the product of two dimensionless ratios, i.e.

$$
\frac{\lambda V_{a w}}{\chi V_{t}}=\frac{\lambda}{\chi} \cdot \frac{V_{a w}}{V_{t}}=\hat{Z}=\frac{1}{2}<1 .
$$

The latter ratios might be assigned a statistical meaning. Thus the quantity $\lambda / \chi>>1$ is taken to be the ratio of auto-wave scale to the minimal possible (lattice) scale or else to the possible number of sites in the system where localized plasticity waves are generated, while the quantity $V_{a w} / V_{t}<<1$ is taken to be a measure for choosing auto-wave rate from the range of possible values, i.e. $0 \leq V_{a w} \leq V_{t}$. In view of the above, the concept of the nature of invariant (1) is formulated as follows. The general variation in the entropy production can be formulated for the deformed system in which auto-waves are generated as the sum of a static (scale) contribution and a kinematic (rate) one, i.e.

$$
\Delta S=\Delta S_{\text {stat }}+\Delta S_{k i n}=\widehat{S}<0,
$$

where $\widehat{S}<0$ expresses a general decrease in the entropy production for the deforming system [6].

Let us evaluate the entropy gain signs in Eq. (10). In view of the above, the static contribution is estimated using the Boltzmann formula for entropy as follows:

$$
\Delta S_{\text {stat }}=k_{B} \ln \frac{\lambda}{\chi}>0,
$$


since $\lambda / \chi>>1$. Here $k_{B}$ is the Boltzmann constant). The rate contribution is estimated as

$$
\Delta S_{k i n}=k_{B} \ln \frac{V_{a w}}{V_{t}}<0,
$$

since $V_{a w} / V_{t}<<1$.

Thus from Eqns. (10-12) follows that

$$
\ln \frac{\lambda}{\chi}+\ln \frac{V_{a w}}{V_{t}}=\widehat{S} / k_{B}<0
$$

and, correspondingly,

$$
\frac{\lambda}{\chi} \cdot \frac{V_{a w}}{V_{t}}=\exp \left(\widehat{S} / k_{B}\right)<1
$$

Apparently,

$$
\hat{Z}=\exp \left(\hat{S} / k_{B}\right)=\frac{1}{2},
$$

hence, $\widehat{S}=k_{B} \ln 1 / 2 \approx-0.7 k_{B}$ as calculated per elementary relaxation act.

\section{Two-component model of the localized plasticity development}

In the conceptual framework used to address autowave formation the basic problem is the nature of self-organization, which manifests itself in the deforming medium as a spontaneous emergence of autowave structure. Physical interpretation of Eqns. (7) and (9) might prove productive for elucidation of the problem. Kadomtsev [7] advanced the idea that a self-organizing system will separate spontaneously into dynamic and information subsystems, interacting with one another.

The working of the proposed model is as follows. In the course of plastic deformation local stress concentrators would form and disintegrate; these are considered as sloweddown shears. Elementary stress relaxation act is due to breaking from a local obstacle, which involves acoustic emission. These acoustic signals will activate other stress concentrators, to so that the same process is repeated over and over again. Thus acoustic emission signals propagating in the deforming medium play the role of information subsystem; dislocation shears are involved in the plastic deformation proper and operate as a dynamic subsystem. The model developed is made up of two components: acoustic emission and dislocation mechanisms of plasticity, which have been studied sufficiently, although in different contexts. The generation of acoustic signals was considered in connection with the initiation of dislocation shears, while the reverse process, i.e. initiation of shears due to acoustic pulses, has not been touched on thus far.

In what follows, the working of the proposed model is assessed. Let acoustic signal propagate in non-uniform dislocation substructure, which forms by deformation and is observable by transmission electron microscopy, e.g. dislocation cell having size $R \approx 0.01$ $\mathrm{mm}$. It was shown earlier [1] that such cell be regarded as acoustic lens, which has focal length, $f_{l}$, given as

$$
f_{l} \approx \frac{R}{V_{t}^{(d e f)} / V_{t}-1},
$$


where the ratio of ultrasound rates, $V_{t}^{(d e f)} / V_{t}$, observed for non-deformed and deformed volumes plays the role of acoustic refractive index. The initiation of plastic deformation is due to the ultrasound waves focusing at distance $\lambda \approx f_{l} \approx 10^{-2} \mathrm{~m}$ from the active localized plasticity nucleus.

Now estimate acoustic emission pulses in terms of energy expenditure required for activating dislocation shears. According to the thermally activated phenomena theory [8], the time needed for dislocations spontaneous depinning from barriers, can be estimated as $\tau_{s p} \approx \omega_{D}^{-1} \exp \left(\frac{U_{0}-\gamma \sigma}{k_{B} T}\right)$. Here $U_{0}-\gamma \sigma=H$ is the process enthalpy, $k_{B}$ is the Boltzmann constant, $\gamma$ is the activation volume of the process and $\omega_{D}$ is the Debye frequency. Generally, $H \approx 1 \mathrm{eV}$ and $\tau_{s p} \approx 10^{-6} \mathrm{~s}$. However, we have $H=U_{0}-\gamma \sigma-\varepsilon_{p h}$ for the case of the inducted depinning. If the phonon energy is given as $\varepsilon_{p h}=\hbar \omega_{D} \approx 0.3 \mathrm{eV}$ that $\tau_{\text {ind }} \approx$ $5 \cdot 10^{-7} \mathrm{~s}<\tau_{s p}$. It is an established fact that the perfect crystal lattice is a source of crystal defects responsible for plastic form changing; therefore, its properties must be taken into account by addressing self-organization processes as well. Hence, the basic premise of the given paper is that the regular features of plastic flow macrolocalization are directly related to the lattice characteristics.

\section{Conclusion}

The elastic-plastic strain invariant has been established experimentally. The analysis of its nature suggests that the development of plastic deformation occurs via auto-wave processes of localized plasticity, involving elastic deformation processes. Hence, the both types of deformation are interdependent, which is suggested by the plastic flow diagram $\sigma(\varepsilon)$. The nature of the elastic-plastic strain invariant is discussed herein. In the given approach, the plastic deformation is regarded as a self-organization process, which occurs in the defect structure of the deformed system and involves a decrease in the production of entropy.

This work was supported by the grant of Russian Scientific Foundation No. 16-19-10025.

\section{References}

1. L.B. Zuev, Autowave Plasticity. Localization and Collective Modes (Fizmatlit, Moscow, 2008)

2. G. Nicolis, I. Prigogine, Exploring Complexity (W. H. Freenan \& Comp, New York, 1989)

3. L.B. Zuev, Phys. Wave Phenom. 20, 166-173 (2012)

4. V.I. Krinsky, Self-Organization: Autowaves and Structures far from Equilibrium (Springer, Berlin, 1984)

5. L.D. Landau, E.M. Lifshitz, Statistical Physics (Butterworth-Heinemann, London, 1980)

6. L.B. Zuev, Bull. Rus. Acad. Sci. (Phys.) 78, 957-964 (2014)

7. B.B. Kadomtsev, Physics - Uspekhi. 164, 449-530 (1994)

8. D. Caillard, J.L. Martin, Thermally Activated Mechanisms in Crystal Plasticity (Elsevier, Oxford, 2003) 